\title{
Prediction of Binding Sites in Protein-Nucleic Acid Complexes*
}

\author{
Namshik Han and Kyungsook Han** \\ School of Computer Science and Engineering, Inha University, Inchon 402-751, Korea \\ han_3567@hotmail.com, khan@inha.ac.kr
}

\begin{abstract}
Determining the binding sites in protein-nucleic acid complexes is essential to the complete understanding of protein-nucleic acid interactions and to the development of new drugs. We have developed a set of algorithms for analyzing protein-nucleic acid interactions and for predicting potential binding sites in protein-nucleic acid complexes. The algorithms were used to analyze the hydrogen-bonding interactions in protein-RNA and protein-DNA complexes. The analysis was done both at the atomic and residue level, and discovered several interesting interaction patterns and differences between the two types of nucleic acids. The interaction patterns were used for predicting potential binding sites in new protein-RNA complexes.
\end{abstract}

\section{Introduction}

A variety of problems concerned with protein-DNA interactions have been investigated for many years, but protein-RNA interactions have been much less studied despite their importance. One reason for this is that only a small number of protein-RNA structures were known. As a result, these structures were generally studied manually on a small-scale. The task of analyzing the protein-RNA binding structures manually becomes increasingly difficult as the complexity and number of protein-RNA binding structures increase. Now that an increasing number of proteinRNA structures are known, there is a need to automatically analyze the interactions involved and to compare them with protein-DNA interactions.

In contrast to the regular helical structure of DNA, RNA molecules form complex secondary and tertiary structures consisting of elements such as stems, loops, and pseudoknots. Generally only specific proteins recognize a given configuration of such structural elements in three-dimensional space. RNA forms hydrogen bonds and electrostatic interactions, and possess hydrophobic groups; it can therefore make specific contacts with small molecules. However, the basis of its interaction with proteins is unclear. In our previous study of protein-RNA complexes, we analyzed the interaction patterns between the protein and RNA at the level of residues and atoms [1]. As an extention of the previous study, we attempted to predict potential binding sites in protein-nucleic acid complexes by analyzing the hydrogen-bonding $(\mathrm{H}-$

* This work was supported by the Ministry of Information and Communication of Korea under grant number 01-PJ11-PG9-01BT00B-0012.

** To whom correspondence should be addressed. email: khan@inha.ac.kr 
bonding) interactions between the amino acids of proteins and the nucleotides of nucleic acids.

\section{Types of Hydrogen Bonding Interactions}

Hydrogen bonds were classified into 3 types: (1) single interactions in which one hydrogen bond is found between an amino acid and a nucleotide, (2) bidentate interactions where an amino acid forms two or more hydrogen bonds with a nucleotide or base-paired nucleotides, and (3) complex interactions where an amino acid binds to more than one base step simultaneously [1]. Our definition of hydrogen bond types is slightly different from that of Luscombe et al. [2]. The latter only analyzed hydrogen bonds between amino acids and bases, whereas we also consider hydrogen bonds with the RNA backbone. Therefore, our study can reveal differences in binding propensities between bases, sugar groups and phosphate groups.

\section{Frameworks}

\subsection{Datasets}

Protein-RNA complex structures were obtained from the PDB database [3]. Complexes solved by X-ray crystallography at a resolution $\leq 3.0 \AA$ were selected. As of September 2002, there were 188 protein-RNA complexes in PDB, and 139 of them were at a resolution $\leq 3.0$ Á. We used PSI-BLAST [4] for similarity searches on each of the protein and RNA sequences in these 139 complexes in order to eliminate equivalent amino acids or nucleotides in homologous protein or RNA structures. 64 out of 139 protein-RNA complexes were left as the representative, non-homologous complexes after running the PSI-BLAST program with an E value of 0.001 and an identity value of $80 \%$ or below. We excluded 13 out of the 64 complexes that have no water molecules or are composed of artificial nucleotides. Table 1 lists the 51 proteinRNA complexes in the final data set. For the dataset of protein-DNA complexes, we used 129 protein-DNA complexes used in the study of Luscombe [2].

Table 1. Protein-RNA complexes in the data set.

\begin{tabular}{lcl}
\hline Molecular & Num. & \multicolumn{1}{c}{ PDB ID } \\
\hline tRNA & 15 & $\begin{array}{l}\text { 1EFW, 1SER, 1C0A, 1QTQ, 1H4Q, 1H4S, 1FFY, 1GAX, 1B23, 1F7U, 1G59, 1IL2, } \\
\text { 1QF6, 2FMT, 1K8W }\end{array}$ \\
\hline mRNA & 1 & 1B7F \\
\hline Ribosome & 6 & 1HC8, 1MMS, 1DFU, 1FEU, 1DK1, 116U \\
\hline Ribozyme & 4 & 1JBR, 1JBS, 1CX0, 1B2M \\
\hline Virus & 12 & 2BBV, 1F8V, 1KNZ, 1E7X, 1HE0, 1HE6, 1HDW, 1ZDH, 1ZDI, 5MSF, 6MSF, 7MSF \\
\hline TRAP & 3 & 1C9S, 1GTF, 1GTN \\
\hline SRP & 4 & 1JID, 1L9A, 1LNG, 1HQ1 \\
\hline Others & 6 & 1FXL, 1G2E, 1DI2, 1EC6, 1KQ2, 1URN \\
\hline
\end{tabular}




\subsection{Hydrogen Bonds}

The number of hydrogen bonds between the amino acids and nucleotides in the protein-RNA complexes was calculated using CLEAN, a program for tidying Brookhaven files, and HBPLUS [5], a program to calculate the number of hydrogen bonds. The hydrogen bonds were identified by finding all proximal atom pairs between hydrogen bond donors (D) and acceptors (A) that satisfy the given geometric criteria. The positions of the hydrogen atoms $(\mathrm{H})$ were theoretically inferred from the surrounding atoms, because hydrogen atoms are invisible in purely X-ray-derived structures. The criteria considered to form the hydrogen bonds for this study were: contacts with a maximum D-A distance of $3.9 \AA$, maximum H-A distance of $2.5 \AA$, and minimum D-H-A and H-A-AA angles set to $90^{\circ}$, where AA is an acceptor antecedent (see Fig. 1).

All protein-RNA bonds were extracted from the HBPLUS output files. There were 1,568 hydrogen bonds in the dataset. We conducted separate experiments in order to compare the properties of single interactions, bidentate interactions and complex interactions, and the results were analyzed for the three types of hydrogen bonds: (1) single interactions, (2) bidentate interactions, and (3) complex interactions.

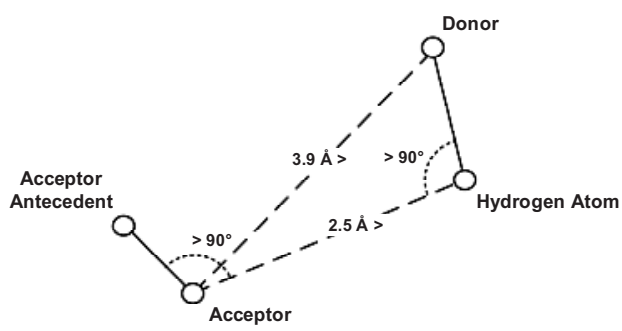

Fig. 1. Angles and distances used in the definition of the hydrogen bonds.

\section{Algorithms}

\subsection{Analysis Algorithm}

As shown in Fig. 2, the analysis algorithm is composed of 4 phases. In phase 1, the algorithm constructs arrays to store the amino acid and nucleic acid sequences, respectively, and classifies hydrogen bonds. These arrays and lists are used to determine interaction types. The algorithm also analyzes whether a nucleotide is paired with other nucleotides. It is essential to discriminate whether binary or multi bond is single interaction or not. So, it is used to classify the interaction types. These processes are the basis of phases 2-4 of the algorithm.

In phase 2, the algorithm investigates the internal hydrogen bond relations of the nucleic acid and records the result of the investigation in a linked-list. It also investigates the hydrogen bonds between the protein and nucleic acid and records this result in a linked-list. These processes are important groundwork for identifying 
binding patterns as they represent the relation between pairs of residues in the form of linked-lists. These are then used in phase 4 to parse the classified interaction types.

In phase 3 , the algorithm classifies the bonding type of each amino acid into unitary, double and multi-bond based on the number of hydrogen bonds between the amino acid and the nucleic acid. It inspects whether the amino acid forms two or more hydrogen bonds with the base or base pair. This is one of the most important processes because it can directly identify the double bond of the bidentate interaction. Since double bonds are abundant, it can eliminate many unnecessary operations.

In phase 4 , the algorithm parses the outcomes of phase 3 to determine binding patterns and numbers of hydrogen bonds involving each region of nucleotides and amino acids. The analysis is done both at the atomic and residue level, and the results help us identify how proteins recognize binding targets, which nucleotides are favored by which amino acids, and their binding sites.

\subsection{Prediction Algorithm}

The prediction algorithm is composed of two phases. In phase 1, it splits unknown protein structure into dices and examines all dices to sort potential binding sites with high probability. Splitting the protein structure requires the coordinate values of all atoms and the center position of every residue. Every PDB file of a structure has the starting coordinate value, which is outside the structure. The algorithm selects the closest residue from the starting coordinates of the structure. It then finds neighbor residues of the closest residue and the residues within a dice.

In phase 2, the algorithm constructs the structure-based residue lists that contain structural information for each dice. It then compares the lists to the nucleic acid sequence to predict potential binding sites using the interaction propensities and patterns. All potential binding sites are examined to predict the best binding site candidate. The structure information was used to eliminate spurious candidates at the last step of prediction. For example, a potential binding site with interaction between sheets in proteins and stems in RNA or DNA is eliminated, since the sheet structure in proteins prefers the loop structure in RNA or DNA. More details are explained in section 5.3.

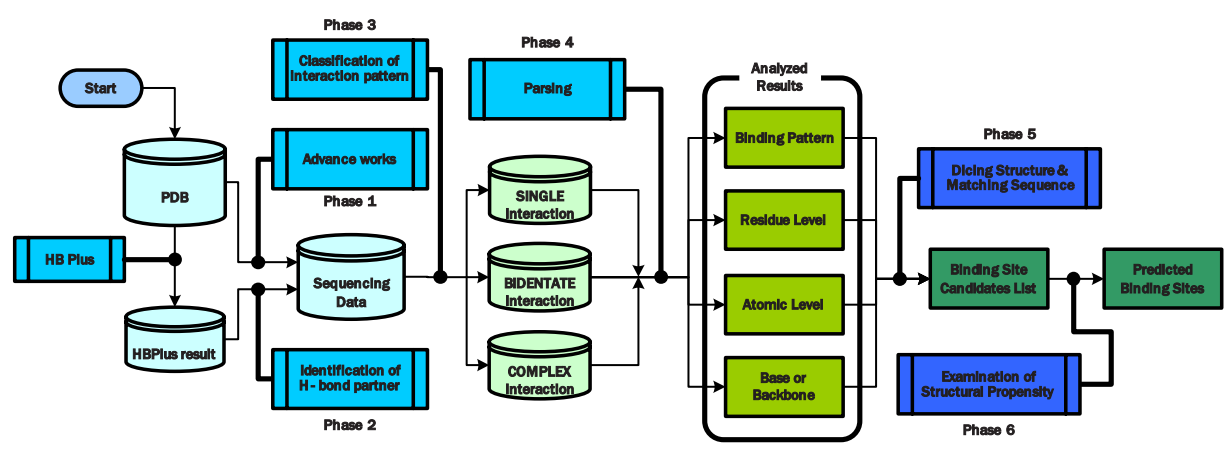

Fig. 2. Sequence for analyzing the protein-nucleic acid complexes and for predicting potential binding sites. 


\section{Results}

\subsection{Differences between DNA and RNA}

In protein-DNA complexes, almost equal numbers of hydrogen bonds were involved in single, bidentate and complex interactions [2]. However, in protein-RNA complexes, $60 \%$ of the hydrogen bonds were found in single interactions.

RNA and DNA were different in their preference for backbone versus base contacts. $32 \%$ of the hydrogen bonds between protein and DNA involved base contacts, compared with $50 \%$ in protein-RNA. This can be explained by the structural difference between RNA and DNA. DNA is a double stranded molecule, and its bases are therefore already involved in hydrogen bonding. Hence, the base region is not as flexible as the backbone and is therefore less able to bind to amino acids. The bases in single-stranded regions of RNA, on the other hand, are quite flexible.

DNA and RNA were also different in their favored amino acids. GLU and ASP have acidic side chain groups, and more frequently hydrogen bind to RNA than to DNA. In protein-RNA complexes, these two amino acids are ranked 5th and 7th, respectively, but in protein-DNA complexes they are ranked 11th and 12th (Table 2). In particular, both GLU and ASP bind very frequently to guanine in the protein-RNA complexes (Table 3). The opposite is the case with GLY and ALA, which bind to DNA more often than to RNA. They rank 10th and 14th, respectively in protein-RNA complexes, but 5th and 9th in protein-DNA complexes. Both GLY and ALA have non-polar side chains, and residues with small side chains bind to double stranded DNA more easily than those with large side chains.

Table 2. Comparison of protein-DNA complexes with protein-RNA complexes in terms of the number of hydrogen bonds in amino acids.

\begin{tabular}{|c|c|c|c|c|}
\hline \multirow{2}{*}{ Rank } & \multicolumn{2}{|c|}{ D N A } & \multicolumn{2}{c|}{ R N A } \\
\cline { 2 - 5 } & Residue & H - bonds & Residue & H - Bonds \\
\hline 1 & ARG & 597 & ARG & 306 \\
\hline 2 & LYS & 293 & LYS & 257 \\
\hline 3 & THR & 292 & SER & 164 \\
\hline 4 & SER & 207 & THR & 151 \\
\hline 5 & GLY & 168 & GLU & 136 \\
\hline 6 & ASN & 167 & ASN & 125 \\
\hline 7 & GLN & 149 & ASP & 116 \\
\hline 8 & TYR & 80 & GLN & 61 \\
\hline 9 & ALA & 71 & TYR & 59 \\
\hline 10 & HIS & 60 & GLY & 40 \\
\hline 11 & GLU & 53 & HIS & 36 \\
\hline 12 & ASP & 19 & PHE & 31 \\
\hline 13 & ILE & 16 & LEU & 19 \\
\hline 14 & CYS & 11 & ALA & 17 \\
\hline$\cdots$ & $\ldots$ & $\ldots$ & $\ldots$ & $\ldots$ \\
\hline 20 & VAL & 3 & MET & 1 \\
\hline
\end{tabular}




\subsection{Interaction Propensities and Patterns in Protein-RNA Complexes}

In bidentate interactions, GLU and ASP mainly bind to guanine whereas THR and LYS generally bind to adenine. This binding preference results in characteristic patterns of binding between the amino acid and nucleotide pairs (Table 3 and Figure $3)$. For example, the binding pattern shown in the GLU-G pair is most common. An exception is LYS: there are 69 hydrogen bonds between LYS and adenine bases, but there is no prominent binding pattern.

In protein-RNA complexes, the side chain of an amino acid binds to the only one base rather than base pairs or base steps. In contrast, there are many hydrogen bonds between a side chain and a base pair or base step in protein-DNA complexes [3]. This difference can again be explained by the structural difference between RNA and DNA.

Table 3. Binding patterns involved in bidentate interactions. The hydrogen bonds donor (D) and hydrogen bond acceptor (A) are indicated in parentheses.

\begin{tabular}{|c|c|c|c|c|c|c|c|c|c|c|}
\hline \multicolumn{3}{|c|}{ ARG } & ASP & ASN & GLU & \multicolumn{3}{|c|}{ GLN } & SER & THR \\
\hline 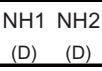 & \begin{tabular}{|cc}
$\mathrm{NE}$ & $\mathrm{NH} 2$ \\
(D) & (D) \\
\end{tabular} & \begin{tabular}{|cc}
$\mathrm{NH} 1$ & $\mathrm{NH} 2$ \\
(D) & (D) \\
\end{tabular} & \begin{tabular}{|cc} 
OD1 & OD1 \\
(A) & (A) \\
\end{tabular} & \begin{tabular}{|cc} 
OD1 & ND2 \\
(A) & (D) \\
\end{tabular} & $\begin{array}{cc}\text { OE1 } & \text { OE2 } \\
(\mathrm{A}) & (\mathrm{A}) \\
\end{array}$ & \begin{tabular}{|cc} 
OE1 & NE2 \\
(A) & (D) \\
\end{tabular} & \begin{tabular}{|cc} 
OE1 & NE2 \\
(A) & (D) \\
\end{tabular} & \begin{tabular}{|cc} 
OE1 & NE2 \\
(A) & (D) \\
\end{tabular} & $\begin{array}{ll}\text { OG } & \text { OG } \\
(\mathrm{A}) & (\mathrm{D}) \\
\end{array}$ & $\begin{array}{l}\text { OG1 } \\
\begin{array}{ll}\text { OG1 } \\
\text { (D) } & \text { (A) } \\
\end{array}\end{array}$ \\
\hline C & $\mathbf{U}$ & G & G & A & G & $\mathbf{U}$ & G & G & A & A \\
\hline $\begin{array}{ll}\text { N3 } & \text { O2 } \\
\text { (A) } & \text { (A) } \\
\end{array}$ & $\begin{array}{ll}\mathrm{O} 2 & \mathrm{O} 2 \\
(\mathrm{~A}) & (\mathrm{A}) \\
\end{array}$ & $\begin{array}{ll}06 & 06 \\
(\mathrm{~A}) & (\mathrm{A}) \\
\end{array}$ & $\begin{array}{ll}\text { N1 } & \text { N2 } \\
\text { (D) } & \text { (D) } \\
\end{array}$ & $\begin{array}{ll}\text { N6 } & \text { N1 } \\
\text { (D) } & \text { (A) } \\
\end{array}$ & $\begin{array}{ll}\text { N1 } & \text { N2 } \\
\text { (D) } & \text { (D) } \\
\end{array}$ & $\begin{array}{ll}\text { N3 } & \text { O4 } \\
\text { (D) } & \text { (A) } \\
\end{array}$ & $\begin{array}{ll}\text { N2 } & \text { O2 } \\
\text { (D) } & \text { (A) } \\
\end{array}$ & $\begin{array}{ll}\text { N2 } & \text { N3 } \\
\text { (D) } & \text { (A) } \\
\end{array}$ & $\begin{array}{ll}\text { N6 } & \text { N1 } \\
\text { (D) } & \text { (A) } \\
\end{array}$ & $\begin{array}{ll}\text { N7 } & \text { N6 } \\
\text { (A) } & \text { (D) } \\
\end{array}$ \\
\hline 11 & 2 & 1 & 12 & 1 & 37 & 2 & 1 & 1 & 1 & 18 \\
\hline
\end{tabular}

(1)

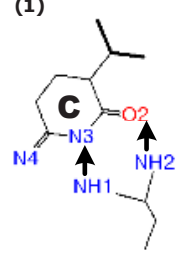

ARG
(2)

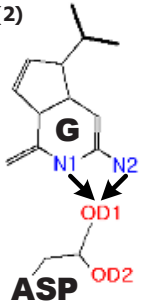

(3)

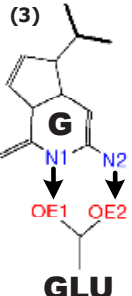

(4)

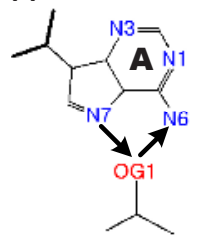

THR

Fig. 3. Frequent binding patterns. Binding patterns (1), (2), (3), and (4) were observed in 11, 12,37 and 18 complexes in the dataset, respectively.

\subsection{Structural Propensities and Binding Sites}

Protein helices bind equally to nucleotide pairs and non-pairs in H-bonding interactions. In contrast, sheets prefer non-pairs to pairs, and turns prefer pairs to nonpairs. Non-pairs have been considered to have high interaction propensity in general, but our study found this is not the case since turns prefer pairs and helices show no preference. In protein-RNA complexes, this implies that sheets prefer to bind to RNA loops and turns prefer to bind to RNA stems [6].

Fig. 4 shows both the known binding sites and the predicted binding sites of the NS5B part of Hepatitis C Virus (HCV) [7], Thermus thermophilus Valyl-tRNA 
synthetase [8] and Escherichia coli Threonyl-tRNA synthetase [9]. Table 4 represents both known and predicted binding sites of the NS5B part of Hepatitis C Virus. The predicted binding sites do not exactly correspond to the known binding sites. However, all predicted binding sites are found near or within the known binding sites, and therefore can reduce the region of potential binding sites effectively.

Table 4. Sequences of the known and predicted binding sites of the NS5B part of Hepatitis C Virus. Residues in red color represent those common in the known and predicted binding sites. Residues in blue represent those in the known sites only.

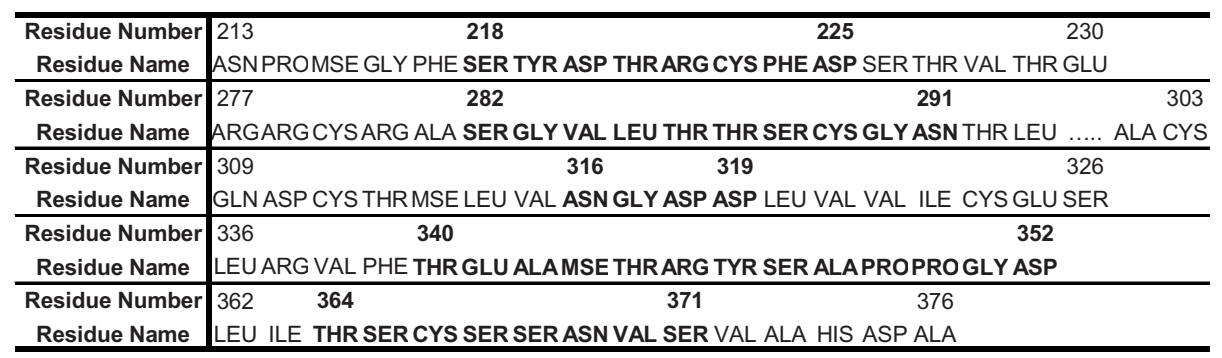

(A)
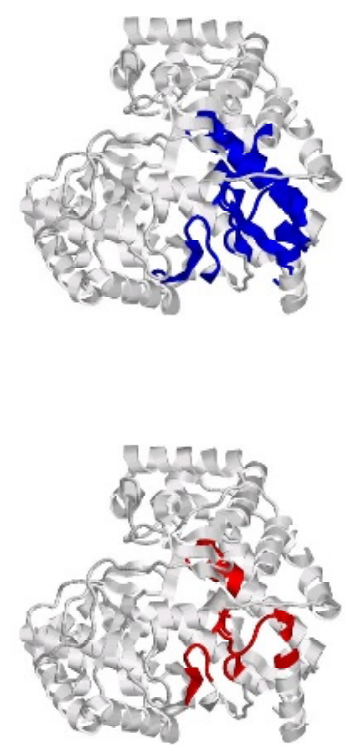

(B)
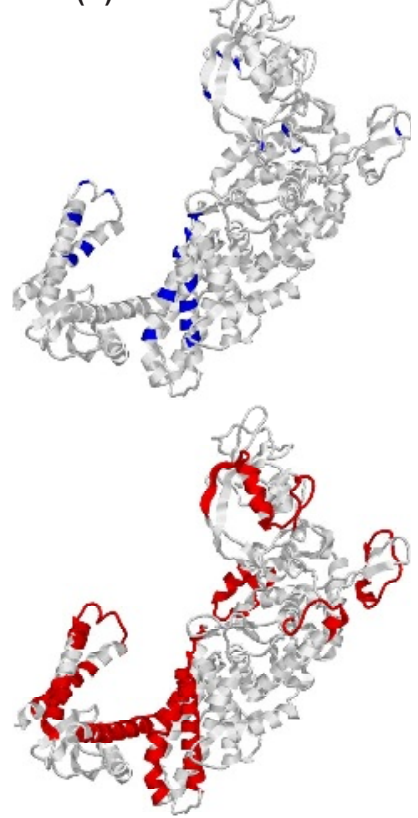

(C)

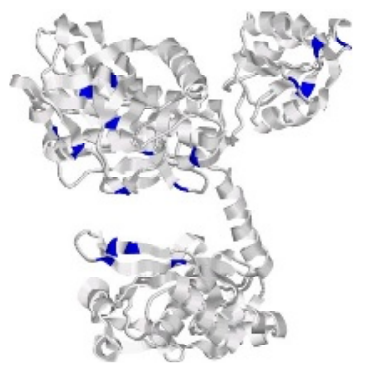

KB

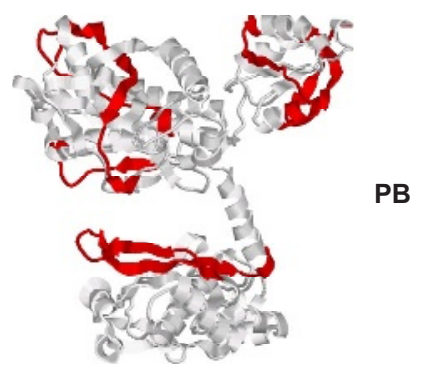

Fig. 4. Known and predicted binding sites of NS5B part of Hepatitis C Virus (A), T. thermophilus Valyl-tRNA synthetase (B), and E. coli Threonyl-tRNA synthetase (C). KB: known binding sites, PB: predicted binding sites. 


\section{Discussion}

We have developed a set of algorithms for analyzing H-bonding interactions and nucleic acids and for predicting potential binding sites between amino acids. This paper presents the results of such an analysis and compares the characteristics of RNA and DNA binding to proteins and prediction results.

The protein-RNA complexes display specific binding patterns. In bidentate interactions in protein-RNA complexes, GLU and ASP overwhelmingly bind to guanine while THR and LYS generally bind to adenine. DNA binds to GLY and ALA preferentially, whereas RNA usually does not binds to them but rather to GLU and ASP. This binding preference results in favored binding patterns. For example, the binding pattern of the GLU-G pair is the most common.

The binding patterns obtained from analyzing $\mathrm{H}$-bonding interactions between amino acids and nucleotides were used to predict potential binding sites of proteinnucleic acid complexes. The binding sites predicted by our algorithm do not exactly correspond to the known binding sites, but it can reduce the region of potential binding sites and the unnecessary experiments. This indicates that prediction was performed in a conservative manner. However, a more rigorous study is required to improve the prediction results for various test cases.

\section{References}

1. Han, N., Kim, H., Han, K.,: Computational Approach to Structural Analysis of ProteinRNA Complexes. Lecture Notes in Computer Science, Vol. 2659 (2003) 140-150

2. Luscombe, N.M., Laskowski, R.A., Thornton, J.M.: Amino acid-base interactions: a threedimensional analysis of protein-DNA interactions at an atomic level. Nucleic Acids Research 29 (2001) 2860-2874

3. Westbrook, J., Feng, Z., Chen, L., Yang, H., Berman, H.M.: The Protein Data Bank and structural genomics. Nucleic Acids Research 31 (2003) 489-491

4. Altschul, S.F., Madden, T.L., Schaffer, A.A., Zhang, J., Zhang, Z., Miller, W., Lipman, D.J.: Gapped BLAST and PSI-BLAST: a new generation of protein database search programs. Nucleic Acids Research 25 (1997) 3389-3402

5. McDonald, I.K., Thornton, J.M.: Satisfying Hydrogen Bonding Potential in Proteins. J. Mol.Biol. 238 (1994) 777-793

6. Kim, H., Jeong, E., Lee, S.-W., Han, K.: Computational analysis of hydrogen bonds in protein-RNA complexes for interaction patterns. FEBS Letters 552 (2003) 231-239

7. Bressanelli, S., Tomei, L., Roussel, A., Incitti, I., Vitale, R.L., Mathieu, M., De Francesco, R., Rey, F.A.: Crystal structure of the RNA-dependent RNA polymerase of hepatitis C virus. Proc. Natl. Acad. Sci. 96 (1999) 13034-13039

8. Fukai, S., Nureki, O., Sekine, S., Shimada, A., Tao, J., Vassylyev, D.G., Yokoyama, S.: Structural Basis for Double-Sieve Discrimination of L-Valine from L-Isoleucine and LThreonine by the Complex of tRNAVal and Valyl-tRNA Synthetase. Cell 103 (2000) 793803

9. Sankaranarayanan, R., Dock-Bregeon, A.-C., Romby, P., Caillet, J., Springer, M., Rees, B., Ehresmann, C., Ehresmann, B., Moras, D.: The Structure of Threonyl-tRNA SynthetasetRNAThr Complex Enlightens Its Repressor Activity and Reveals an Essential Zinc Ion in the Active Site. Cell 97 (1999) 371-381 